\title{
Milliliter per Breath
}

National Cancer Institute

\section{Source}

National Cancer Institute. Milliliter per Breath. NCI Thesaurus. Code C73749.

A dose calculation unit expressed in milliliter(s) per inspiration or expiration of breath. 